\title{
Gratitude Expressions Improve Teammates' Cardiovascular Stress Responses
}

\author{
Yumeng Gu${ }^{1}$, Joseph M. Ocampo ${ }^{2}$, Sara B. Algoe ${ }^{3}, \&$ Christopher Oveis $^{1}$ \\ ${ }^{1}$ University of California, San Diego \\ ${ }^{2}$ University of California, Berkeley \\ ${ }^{3}$ University of North Carolina at Chapel Hill
}

\section{Author Note}

The authors thank Melody Chen, Tayler Bergstrom, Hee Yeon Hwang, Isaac Raymundo, Giovanna Sun, and Salina Yun for assistance with this work. This work was supported by a grant from the John Templeton Foundation (\#56458) to the third and fourth authors. Correspondence concerning this article should be addressed to Yumeng Gu at yumeng.gu@ rady.ucsd.edu or Christopher Oveis at oveis@ucsd.edu. The experiment reported in this article was not formally preregistered. Data and a preprint of the manuscript are available on OSF at https://osf.io/3rcpd/ and PsyArXiv at https://psyarxiv.com/ur5pg. Authors declare no conflict of interest. 


\begin{abstract}
Gratitude expressions play a key role in strengthening relationships, suggesting gratitude might promote adaptive responses during teamwork. However, little research has examined gratitude's impact on loose tie relationships (like coworkers), and similarly little research has examined how gratitude impacts physiological stress responding or biological responses more generally. The present research uses an ecologically valid, dyadic teamwork paradigm to test how gratitude expressions impact in vivo physiological challenge and threat stress responding, assessed via a challenge-threat index composed of cardiac output and total peripheral resistance. Compared to a control condition, teammates $(N=190)$ who were randomly assigned to a gratitude expression manipulation showed improved biological challenge-threat responses while jointly completing an acutely stressful collaborative work task (developing a product pitch), and later while completing an individual performance task (pitching the product). During the collaborative task, gratitude expressions buffered against threat responses; during the individual task, gratitude expressions amplified challenge responses. Analyses of CO and TPR aided in determining how cardiac outflow versus vascular constriction/dilation contributed to these effects. The finding that gratitude expressions promote adaptive biological responding at the dyadic level contributes to a growing literature on the social functions of positive emotions and gratitude, specifically. The present results also have wider implications for physiological stress in performance tasks and suggest that workplace gratitude interventions can promote adaptive stress responding in teams.
\end{abstract}

Keywords: gratitude, challenge, threat, teams 


\section{Gratitude Expressions Improve Teammates' Cardiovascular Stress Responses}

Over the past 15 years, the accumulation of evidence for the central and largely beneficial role of the emotion of gratitude in social life has accelerated across psychological and organizational sciences. Researchers have documented that gratitude influences a wide variety of behavioral and phenomenological outcomes, such as affiliative behavior, perceptions of partner responsiveness, and personal and relational well-being, largely examining these effects between romantic partners or strangers (see Algoe, 2012, 2019). Despite this ever-growing body of evidence, two important areas of inquiry have been relatively neglected: the interpersonal dynamics of gratitude between loose ties, like acquaintances or co-workers, and the potentially beneficial ways that these dynamics influence biological outcomes when members of the dyad interact. Here, we contribute substantially in these two domains by experimentally manipulating gratitude between loose-tie teammate dyads, and testing the teammates' in vivo stress responses during ecologically valid stressful teamwork.

Building on a substantial body of evidence that the momentary emotional response of gratitude to another person for their kind actions helps promote a high-quality, communal relationship between the grateful person and their benefactor (see review in Algoe, 2012), many researchers have focused on expressed gratitude as a behavioral mechanism that facilitates that dyadic process (e.g., Williams \& Bartlett, 2015). One nice feature of this rapidly expanding body of literature is that the evidence often comes from studies involving both members of the dyad (e.g., Algoe, Fredrickson, \& Gable, 2013; Brady et al., 2020; Leong et al., 2020; Park et al., 2019); as one example, couples randomly assigned to express gratitude to one another over a month-long period reported greater daily adaptation to change as well as positive mood compared to couples in a control condition (Algoe \& Zhaoyang, 2016). At the same time, most 
of these data come from just one type of relationship that is important to everyday liferomantic — whereas other important types of relationships deserve increased attention.

The present work focuses on the dyadic consequences of gratitude expressed between members of loose-tie relationships (university suitemates) working together on a stressful motivated performance task conducted under time- and social evaluative-pressure. This research holds meaningful implications for organizations, and particularly teams, which involve loose ties who often work together under acutely stressful conditions to accomplish joint goals. Gratitude expressions within work environments may be a key to building relationships, binding together teammates, and potentially making joint tasks seem less threatening. In building relationships, gratitude expressions could promote more efficient team stress responses by enhancing perceived personal or social resources or by decreasing the perceived demands of stressful tasks. Consistent with this view, for example, thinking of a supportive friend caused individuals to perceive their environment as less demanding and view challenges in a more moderate way (Schnall et al., 2008). Previous research on social support also found beneficial influences of received support on physiological reactivity during stressful scenarios and links to improved physical health outcomes (Gerin et al., 1992; Krause, 2001; Lepore, Allen, \& Evans, 1993; Shaw et al., 2004). People spend a third or more of their daily lives at work; thus, understanding how gratitude can shape stress responding during teamwork is a critical topic of examination. But, thus far, no dyadic data exist to test these propositions; the present research addresses them directly.

\section{Gratitude Expressions and Challenge/Threat Responses}

A second critical advance of this research is examining the biological consequences of expressed gratitude. Our approach is guided by the biopsychosocial (BPS) model of challenge and threat, which provides a framework for understanding how appraisal processes impact 
responses to acute stress (for reviews, see Blascovich \& Mendes, 2010; Jamieson et al., 2013; Mendes \& Park, 2014). When people appraise that the demands of a task exceed their own resources to complete the task, they are likely to experience a threat response, marked by less efficient cardiovascular activation. In contrast, when people appraise that their resources exceed the demands of the task, they are likely to experience a challenge response, marked by more efficient cardiovascular activation. The BPS model of challenge and threat specifies the underlying psychological mechanisms of stress responses in performance contexts. Specifically, the psychological mechanism underlying the BPS model is the perceptions of "demands" and “resources". Demands consist of perception of uncertainty, danger, and/or effort. Motivated performance situations, such as group projects, are stressful in that they contain important yet uncertain consequences.

Determining whether gratitude expressions impact challenge and threat responses is important because of the focal connection between challenge and threat responding and the quality of task performance (e.g., Moore et al., 2012; Seery et al., 2010), and because physiological patterns of challenge and threat have important downstream consequences. For example, threat responses impair decision making (Kassam et al., 2019), whereas challenge responses are associated with better performance in cognitive and motor tasks (Turner et al., 2012). Over the long term, threat responses are associated with elevated risk for cardiovascular disease, less effective immune response, and cognitive ability impairments (e.g., Jefferson et al., 2010; Matthews et al., 1997). Moreover, challenge and threat responses have been used to conceptualize and assess resilience — defined as adaptation to potentially stressful experiencesduring acute and mundane stressors (Seery, 2011, 2013). 
Importantly, patterns of challenge and threat can be reliably assessed at a biological level (Blascovich \& Tomaka, 1996; Blascovich \& Mendes, 2000) by focusing on two key outcomescardiac output ( $\mathrm{CO}$; the amount of oxygenated blood pumped from the heart to the periphery) and total peripheral resistance (TPR; constriction of the vasculature) - or the compensatory relationship between these two measures. Some research has suggested that CO and TPR should be assessed separately. In this approach, challenge responses are thought to involve increased $\mathrm{CO}$ alongside decreased TPR, whereas threat responses involve unchanged or decreased CO alongside unchanged or increased TPR (e.g., Blascovich \& Tomaka, 1996; Blascovich \& Mendes, 2000; Blascovich, Seery, Mugridge, Norris, \& Weisbuch, 2004; Chalabaev, Major, Cury, \& Sarrazin, 2009; Mendes, Blascovich, Major \& Seery, 2001; Uphill, Rossato, Swain, \& O'Driscoll, 2019). While the present research presents individual analyses of CO and TPR for comparability with previous research in this tradition, this approach has some limitations. First, it ignores that challenge and threat responses can manifest in various ways-due to increased or reduced cardiac outflow, changes in vascular constriction/dilation, or both (Griffin \& Howard, 2021, in press). Second, and relatedly, analyzing CO and TPR separately ignores the compensatory relationship between these two variables: When one of CO or TPR changes, the other typically changes in complementary fashion to promote healthy blood flow and homeostasis; however, this is not always the case (Griffin \& Howard, 2021). Third, it is desirable to have a single measurement to determine if a challenge or threat response occurred, as well as the size of the challenge or threat effect. This is particularly the case for threat responses, which, as noted, sometimes involve changes in $\mathrm{CO}$ and TPR, but sometimes do not. Thus, single measures incorporating both CO and TPR are useful for capturing challenge and threat responses (Griffin \& Howard, 2021, in press; Seery et al., 2010). Within the literature on the 
biopsychosocial model, a challenge-threat index (computed by subtracting standardized TPR from standardized CO) has played this role (e.g., Blascovich, et al., 2004; Hangen et al., 2019; Seery et al., 2010). In the present paper, we thus compute and focus on a challenge-threat index as our focal assessment of challenge and threat responding. Here, we offer a methodological contribution by offering a new computation of the challenge-threat index that provides a meaningful scale zero point (as we show in the Supplemental Material, between-condition statistical comparisons are identical when using the typical calculation). To provide (a) comparability with related research and (b) additional information about the challenge or threat response, we additionally provide data and analyses for $\mathrm{CO}$ and TPR responses individually.

The present study advances the literature on gratitude with novel contributions. In research with individuals, few studies have found physiological consequences of gratitude — on markers of inflammation and heart rate variability (Redwine et al., 2016), and on arousal (Drążkowski, Kaczmarek, \& Kashdan, 2017). Critically, both used gratitude journaling paradigms rather than gratitude expression; neither investigated stress-related physiological responses or used a dyadic paradigm. Only one correlational study has demonstrated an association between individuals' state gratitude and systolic blood pressure reactivity (Ginty et al., 2020). For the first time, the present research examines the dyadic consequences of gratitude expression on stress-relevant physiological responses during ecologically valid stress tasks. It represents a leap forward in our understanding of the potential implications of gratitude in teamwork specifically, and in ongoing relationships of everyday life, more generally.

\section{Current Research}

The present study examines the dyadic, biological consequences of gratitude expressed between people in a loose-tie relationship. After one member is randomly assigned to a gratitude 
or neutral expression, partners complete a stressful, ecologically valid teamwork paradigm involving two sequential tasks: a collaborative work task (to assess effects when partners are actively working together) and an individual performance task (to assess whether effects persist after the partners are no longer actively interacting). We predicted that gratitude expressions, which have been shown to build relationships, would promote improved challenge-threat physiological responses in teams. Due to gratitude's dyadic interpersonal consequences (Algoe \& Zhaoyang, 2016), we had no expectation of differences between expressers and receivers, so we analyzed the data focusing on the dyadic-level condition effect on individuals, using multilevel models.

\section{Method}

Sample size determination. An a priori power analysis was used to determine sample size. There is no previous research investigating gratitude and challenge and threat physiological measurement. Therefore, we based our power analysis on previous work on challenge and threat responses with in vivo cardiovascular measures in dyads (Peters et al., 2014), suggesting an anticipated effect size of $d=0.59$. Given the complexity of estimating power for multilevel analysis, we more conservatively used effect size of $d=0.5$. In Optimal Design Software (Raudenbush et al., 2011), an a priori power analysis determined that 75 dyads would be necessary to achieve 0.8 power. Anticipating the potential for data loss, we decided to recruit 100 dyads.

Participants. Two hundred undergraduates from the University of California, San Diego participated in dyads, receiving \$24 each as a part of a larger study on gratitude expressions (Study approved by the UCSD Human Research Protections Program under Project 151219S). Each dyad consisted of same-gender, first-year students who had been living together as 
suitemates for approximately four months. Ten participants were excluded due to unusable physiological data and two were excluded due to experimenter error. The final sample $(N=190$; 144 women, 46 men; $M_{\text {age }}=18.1, S D_{\text {age }}=1.10$, Range $=18-20 ; 112$ Asian $/$ AsianAmerican/Pacific Islander, 20 Hispanic/Latino, 18 White/Caucasian, 1 Black/African-American, 37 other) consisted of 47 control and 48 gratitude dyads.

Design. Each dyad was randomly assigned to the control or gratitude condition. Within each condition, one participant was randomly assigned to be the expresser, who would express gratitude or a control expression to the receiver; the other participant was randomly assigned to be the receiver, who would listen to the expresser and respond as they would in a normal conversation.

Procedure (see Figure 1 for an overview). In separate testing rooms, two participants completed intake questionnaires and had physiological sensors attached. After acclimating to the lab for 5 minutes, baseline physiological recordings were collected for 5 minutes while participants were seated and resting alone in the room. Next, participants completed self-report measures on a tablet computer and selected the topic they might discuss during the initial conversation and completed the brainstorm portion of the experimental manipulation (see Supplemental Material for details). Members of the dyad were then brought together in a large testing room and completed the gratitude or control expression task (see details in "Experimental manipulation" section). Finally, all participants completed the collaborative work task followed by the individual performance task, during which we assessed challenge- and threat-relevant physiological responses. 
(1)

(2)

(3)

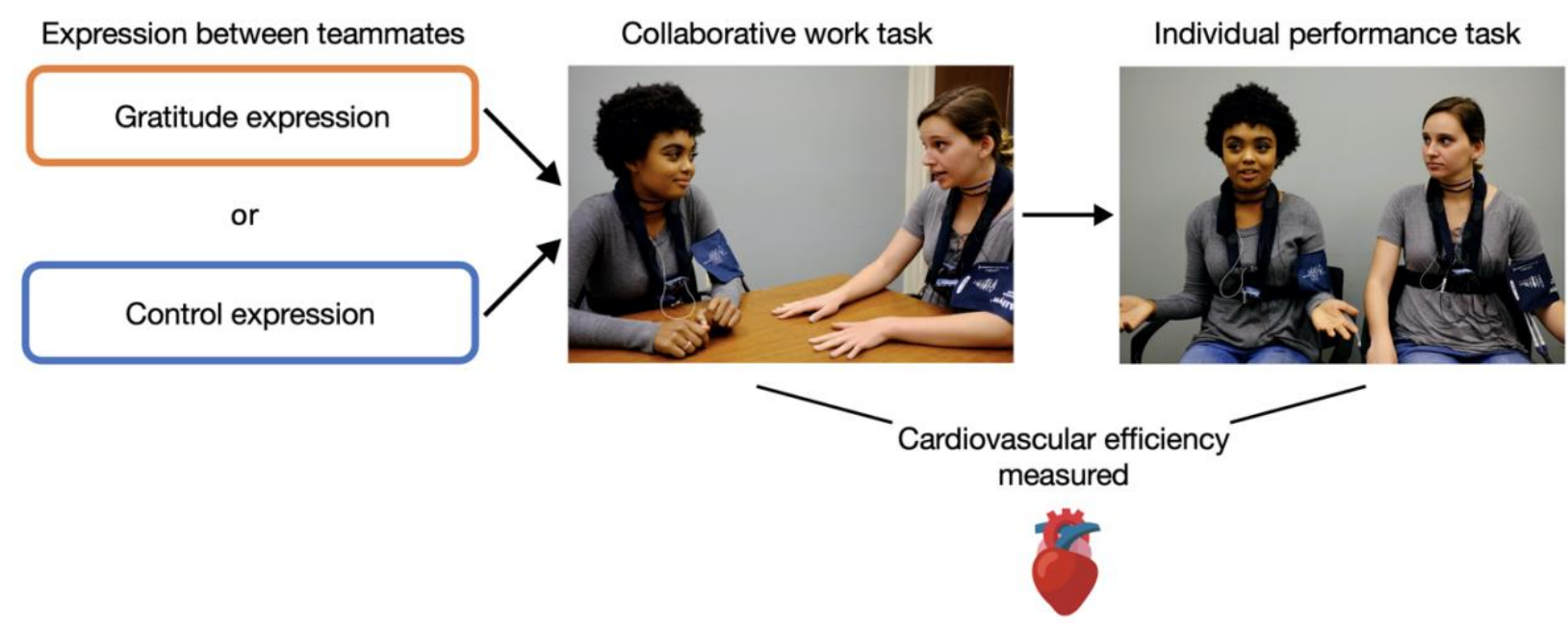

Figure 1. Procedure overview. 1) Dyads first completed a gratitude or control expression task. 2) The teammates next completed the collaborative work task during which they designed a product, marketing plan, and pitch. 3) Each teammate then completed the individual performance task by presenting their part of the product pitch to evaluators.

Experimental manipulation. When completing questionnaires alone, all participants were asked to generate a topic they might discuss in an upcoming conversation. In the gratitude condition, the expresser selected the topic they might discuss with their teammate by writing about an action by their partner (the other participant) for which they felt grateful (Algoe, Fredrickson, \& Gable, 2013). The expresser wrote down what their partner did to cause them to feel gratitude, and why the behavior was especially great and praiseworthy. All other participants (i.e., the receiver in the gratitude condition and both participants in the control condition) wrote about ordinary aspects of an average day (e.g., what their course schedule was like, what they did between classes). 
When members of the dyad reunited, the experimenter revealed the roles to the participants. The expresser then discussed the events they wrote about, either gratitude or control depending on the condition, while both participants were seated at a table for a maximum of two minutes. During this time, the receiver listened and responded naturally, engaging in the topic as much or as little as they would in a normal conversation. Immediately after the conversation, the expresser and the receiver were asked to assess how grateful they felt and their partner appeared during the conversation on a 1 (not at all) to 5 (very much) scale, along with a variety of other emotions.

Collaborative work task and individual performance task. Challenge- and threatrelevant physiological responses were assessed during a collaborative work task (6 minutes) and then during an individual performance task (3 minutes per participant; see Oveis et al., 2020 for procedural details). Both tasks were designed to produce acute stress, and the individual performance task bears resemblance to the Trier Social Stress Task (Kirschbaum et al., 1993). During the collaborative task, the teammates together designed a bicycle, a marketing plan, and a product pitch while seated together at a table. During the individual task, the teammates took turns delivering their individual parts of their product pitch to a pair of evaluators who withheld verbal and nonverbal feedback. To ensure that participants would work together during the collaborative task, the teammates did not learn which teammate had been randomly assigned to complete part one versus part two of the individual task until after the collaborative task had concluded. To incentivize task engagement and heighten acute stress, participants were informed that the best team would receive $\$ 200$.

Physiological measures. During baseline, collaborative work, and individual work, electrocardiography (ECG) signals were collected using a modified Lead II configuration with 
electrodes placed on the torso. Impedance cardiography (ICG) signals were obtained using band electrode that encircled the neck and torso. ECG and ICG signals were sampled at $1 \mathrm{KHz}$ and integrated with a Biopac MP150 (Biopac System Inc., Goleta, CA), processed into 30-second segments, and ensembled into segment averages using Mindware software (IMP v. 3.1.16; Mindware Technologies, Gahanna, $\mathrm{OH}$ ). Blood pressure readings were obtained using a Colin BP-8800 ${ }^{1}$ (Colin Medical Instruments, San Antonio, TX) from the brachial artery of the nondominant arm. Physiological reactivity scores for the collaborative and individual tasks were computed by subtracting averaged baseline scores from averaged collaborative and individual task scores, respectively.

As indicated above, our analyses focused on the challenge-threat index, with additional information provided from its constituent measures of cardiac output $(\mathrm{CO})$ and total peripheral resistance (TPR). Pre-ejection period (PEP) was used as an indicator of sympathetic arousal during the tasks. Blood pressure data are provided in the Supplemental Material. CO is a measure of the amount of blood ejected from the heart per minute. TPR is a measure of vascular resistance to blood flow to the periphery, and was calculated as mean arterial pressure / $\mathrm{CO} * 80$ (Blascovich, Mendes, Vanman, \& Dickerson, 2011). Challenge-threat index was calculated using a similar approach to Blascovich et al. (2004), Hangen et al. (2019), and Seery et al. (2010): They z-scored reactivity measures of CO and TPR, then subtracted TPR from CO. This approach advantageously creates a useful continuous index for relative comparisons between experimental conditions. However, the approach is problematic for interpreting absolute challenge- and threatrelevant responding when not comparing between conditions because the $\mathrm{z}$-scoring renders the

\footnotetext{
${ }^{1}$ The Colin BP-8800 was calibrated and met the performance requirements of the AAMI (Association for the Advancement of Medical Instrumentation). However, we note that this blood pressure monitor failed clinical validation in a study by Naschitz et al. (2000).
} 
zero-point of the scale meaningless. Here, we offer a modification to this calculation in order to preserve a meaningful zero point on the challenge-threat index, in which values greater than zero indicate a challenge response and values less than zero indicate a threat response: In our calculation, we follow a similar procedure, but only partially standardize the scores. We divide reactivity scores for $\mathrm{CO}$ and $\mathrm{TPR}$ by their $\mathrm{SD}$, but do not mean-center them, using the following formula:

Challenge-threat index $=\left(\mathrm{CO}\right.$ reactivity $/ \mathrm{SDCO}_{\mathrm{C}}$ ractivity $)-(\mathrm{TPR}$ reactivity $/ \mathrm{SDTPR}$ reactivity $)$

Using this calculation, zero indicates no particular direction for cardiovascular efficiency (i.e., no change or a mixed response), negative values reflect reduced cardiovascular efficiency, and positive values reflect greater cardiovascular efficiency. Across this dataset, our approach correlated $r>.99$ with the typical calculation of challenge-threat index (Blascovich et al., 2004; Hangen et al., 2019; Seery et al., 2010), and produces identical results when comparing between conditions (see Supplemental Material). Thus, the challenge-threat index provided a single target variable indicating whether relative differences in challenge- and threat-patterned cardiovascular responses would be observed in the gratitude vs. control conditions. The CO and TPR scores, in turn, provided further information about how cardiac outflow and vascular dilation/constriction, individually, contributed to these responses.

Experienced gratitude. Participants rated how "grateful/appreciative/thankful" they and their teammate felt during the conversation on 1 (not at all) to 5 (very much) scale.

Experienced positive affect. Participants rated positive emotions felt during the conversation on 1 (not at all) to 5 (very much) scales, including “amused/having fun”, 
"love/closed/trust", "happy/pleased/joyful”, "proud/good about myself”, “energized/excited/enthusiastic", "admiration/inspired by others", and "compassionate/sympathetic". Positive affect (PA) was retained as the average of these positive emotions $(\alpha=0.84)$.

\section{Results}

Manipulation check. The gratitude condition successfully produced gratitude in the expresser, as felt by the expresser and perceived by the receiver. Expressers in the gratitude condition $(M=4.52, S D=0.62)$ felt significantly more grateful during the conversation than expressers in the control condition $(M=3.38, S D=1.14, F(1,93)=37.39, p<.001,95 \% \mathrm{CI}$ $[0.77,1.52], d=1.26)$. Receivers in the gratitude condition rated their expresser counterparts as experiencing more gratitude $(M=4.49, S D=0.72)$ than receivers in the control condition $(M=$ $3.50, S D=0.88, F(1,93)=36.18, p<.001,95 \% \mathrm{CI}[0.77,1.72], d=1.25)$.

\section{Do teammates show improved biological challenge-threat responses after a}

gratitude expression? Our focal analyses examined whether team members showed more efficient stress responses following a gratitude expression from one teammate to another. To examine potential data non-independence within dyad, we built a two-level multilevel model nesting participant within dyad using the nlme package (v3.1-141, Pinheiro et al., 2019) in R (R core team, 2019). Significant dyad-level variance was observed for PEP $\left(\chi^{2}(1)=5.66, p=.017\right)$. Although dyadic variance was not significant for challenge-threat index $\left(\left(\chi^{2}(1)=2.07, p\right.\right.$ $=.150)$, the $95 \%$ confidence interval showed a non-zero random effect estimation $[0.1 .31,1.77]$. We account for the non-independence in all models to keep them consistent between DVs and to best represent the structure of the experimental design. Therefore, to account for this 
interdependence in the data, we conducted all analyses using two-level nested models of participant within dyad.

Baseline. No baseline physiological differences were observed between the two conditions $(\mathrm{PEP}: F(1,92)=0.43, p=.512$; CO: $F(1,92)=0.70, p=.403$; TPR: $F(1,92)=0.60, p$ $=.439)$.

Collaborative work task: PEP. As intended, the collaborative task elicited sympathetic arousal, indicating that the task was demanding: Collapsing across conditions, participants showed a significant decrease in PEP during the collaborative task compared to baseline $(M=$ $8.82, S D=11.94 ; t(174)=-9.77, p<.001,95 \%$ CI $[-10.60,-7.04], d=-1.48)$. As expected, PEP reactivity did not differ between the gratitude $(M=-9.51, S D=11.91)$ and control conditions $(M$ $=-8.14, S D=11.99), F(1,85)=0.57, p=.452$.

Collaborative work task: Challenge-threat index, CO, and TPR. Collapsing across conditions, participants showed significantly more threat-patterned physiological responses during the collaborative work task compared to baseline, $t(173)=-2.16, p=.032,95 \% \mathrm{CI}[-0.52$, -0.02], as indicated by the challenge-threat index. Participants did not significantly differ in CO during the collaborative work task compared to baseline $(M=0.14, S D=1.66), t(174)=$ $1.13, p=.262$. Participants showed significantly higher TPR during the collaborative work task compared to baseline $(M=74.44, S D=196.45), t(173)=5.00, p<.001,95 \%$ CI $[45.05,103.83]$.

We tested our focal hypotheses by examining how the gratitude expression manipulation impacted the challenge-threat index during stressful collaborative work. For our focal test, we used a mixed effect model to test the fixed effect of condition on the challenge-threat index, with a random intercept for dyads. As predicted, gratitude expressions $(M=0.05, S D=1.95)$ produced less threat-patterned cardiovascular responding compared to the control condition $(M=$ 
$-0.59, S D=1.22$ ), as measured by challenge-threat index reactivity, $F(1,88)=6.00, p=.016, b$ $=.64,95 \%$ CI $[0.12,0.64], d=.52$ (see Figure 2, Panel A). Whereas the control condition showed threat-patterned responding on the challenge-threat index that was significantly different from zero, $b=-0.59, t(88)=-3.19, p=.002,95 \%$ CI $[-0.96,-0.22]$, the gratitude condition did not show threat-patterned responding, $b=0.05, t(88)=0.27, p=.786,95 \%$ CI $[-0.32,0.42]$. 

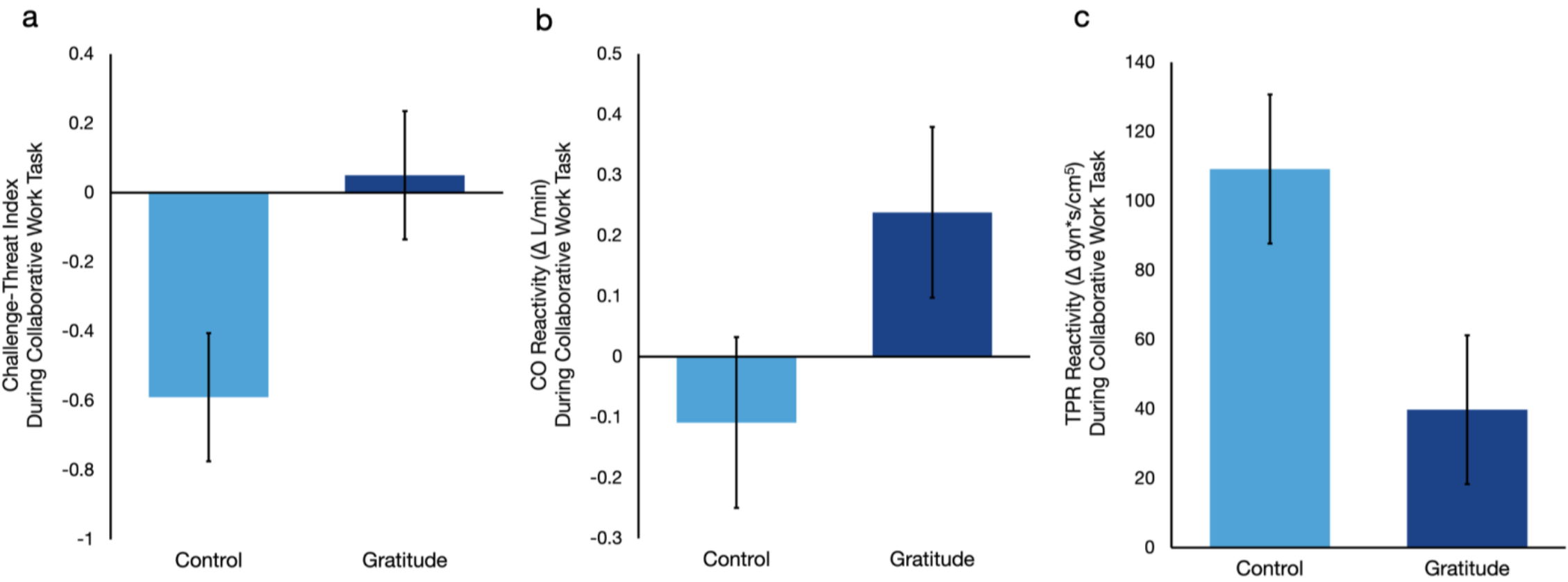

Figure 2. When one member of a team expressed gratitude to the other prior to engaging in stressful collaborative work, the team members were buffered from inefficient (threat-patterned) cardiovascular responding compared to controls, as indicated by the challenge-threat index (Panel A). Gratitude expressions produced marginally improved CO (Panel B) and significantly improved TPR (Panel C). Error bars represent one standard error. 
Decomposing this index into CO and TPR, we used mixed effect models to test the fixed effect of condition on $\mathrm{CO}$ and TPR, with a random intercept for dyads. Gratitude expressions $(M$ $=0.39, S D=2.03)$ produced marginally higher $\mathrm{CO}$ reactivity compared to the control condition $(M=-0.11, S D=1.13), F(1,88)=3.61, p=.060,95 \%$ CI $[-0.02,1.04], d=.41$ (see Figure 2, Panel B). We next examined whether the gratitude and control conditions individually differed from zero change using the multiple intercept form of the model: Whereas the gratitude condition produced challenge-patterned $\mathrm{CO}$ responding that was significantly different from zero $(t(88)=2.11, p=.038,95 \% C I[0.02,0.78])$, control condition CO responding did not differ from zero $(t(88)=-0.576, p=.566)$.

Gratitude expressions $(M=39.84, S D=216.32)$ generated significantly lower (less threat-patterned) TPR reactivity relative to the control condition $(M=109.05, S D=168.56)$, $F(1,88)=5.22, p=.025,95 \%$ CI $[-129.78,-9.06], d=.49$ (see Figure 2, Panel C). Whereas the control condition produced threat-patterned TPR reactivity that significantly differed from zero $(t(88)=5.09, p<.001,95 \%$ CI $[161.38,217.07])$, gratitude condition TPR reactivity marginally differed from zero $(t(88)=1.85, p=.067)$.

Collaborative Work Task: Controlling for PA. One possibility is that speakers' positivity, rather than the expression of gratitude specifically, could account for the effects of condition on challenge and threat. To account for this possibility, we conducted an ANCOVA to control for speakers' positive affect during manipulation, and did not find that PA could account for the observed effects. Gratitude dyads continued to produce more challenge-patterned physiological responses compared to the control condition, as measured by challenge-threat index reactivity, $F(1,87)=6.34, p=.014,95 \% \mathrm{CI}[0.15,1.23], d=.54$. Gratitude dyads also now showed significantly higher CO reactivity, $F(1,87)=5.19, p=.025,95 \%$ CI $[0.08,1.18], d=.49$, 
and continued to show significantly lower TPR reactivity, $F(1,87)=4.06, p=.047,95 \%$ CI [127.28, -0.88$], d=-.43$, compared to control dyads when controlling for speakers' PA.

Individual performance task: PEP. As intended, the individual task elicited sympathetic arousal, indicating that the task was demanding. Collapsing across conditions, participants showed a significant decrease in PEP during the individual task compared to baseline $(M=$ 22.11, $S D=16.64 ; t(171)=-17.42, p<.001,95 \%$ CI $[-24.62,-19.61], d=-2.66)$. As expected, PEP reactivity did not differ between the gratitude $(M=-22.84, S D=18.75)$ and control conditions $(M=-21.39, S D=14.31, F(1,87)=0.32, p=.570$.

Individual performance task: Challenge-threat index, CO, and TPR. Collapsing across conditions, participants showed significantly more threat-patterned physiological responses during the individual performance task compared to baseline $(M=0.69, S D=1.60), t(155)=$ $5.39, p<.001$. Participants showed significantly higher CO during the individual performance task compared to baseline $(M=1.40, S D=2.30), t(171)=7.96, p<.001,95 \%$ CI $[1.05,1.74], d$ $=1.22$. Participants showed significantly lower TPR during the individual performance task compared to baseline, $M=-36.12, S D=205.97, t(155)=-2.19, p=0.03,95 \%$ CI [-68.69, -3.54$]$, $d=-0.35$.

For our focal test, we used a mixed effect model to test the fixed effect of condition on the challenge-threat index, with a random intercept for dyads. As predicted, gratitude expressions $(M=0.98, S D=1.84)$ produced more challenge-patterned cardiovascular responding compared to the control condition $(M=0.38, S D=1.22)$, as measured by challenge-threat index reactivity, $F(1,85)=5.60, p=.020,95 \%$ CI $[0.10,1.10], d=.52$ (see Figure 3$)$. Individually, both the gratitude condition, $b=0.98, t(88)=5.58, p<.001,95 \% \mathrm{CI}[0.63,1.32]$, and control condition, 
$b=0.38, t(88)=2.09, p=.039,95 \%$ CI $[0.02,0.74]$, showed challenge-threat index values significantly greater than zero. 

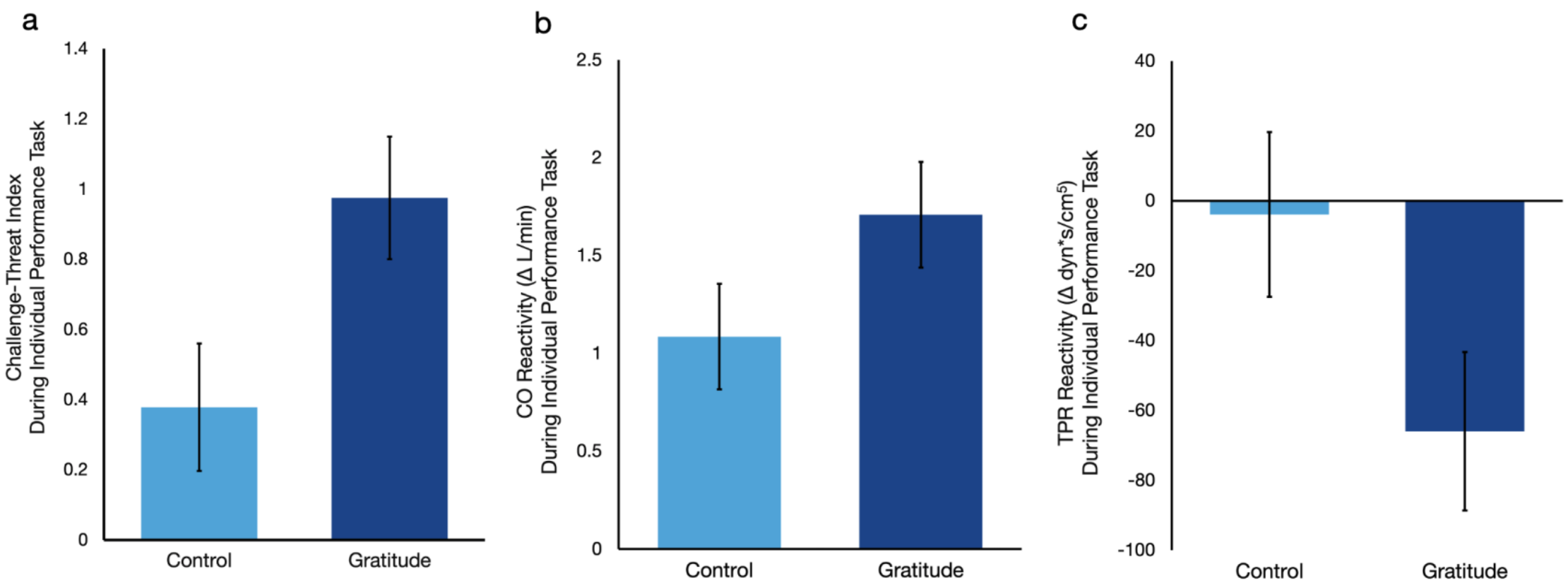

Figure 3. The individual performance task occurred temporally further from the gratitude manipulation and when teammates were no longer actively engaged with one another. Gratitude expressing teams showed more challenge-patterned cardiovascular responding compared to controls, as indicated by the challenge-threat index (Panel A). Both control and gratitude teams showed challengepatterned CO reactivity and did not differ from each other (Panel B). Gratitude expressions produced marginally improved TPR reactivity (Panel C). 
The gratitude $(M=1.71, S D=2.71)$ and control $(M=1.09, S D=1.76)$ conditions did not significantly differ in $\mathrm{CO}$ reactivity, $F(1,87)=2.66, p=.106$. Both the gratitude $(t(87)=6.32, p$ $<.001,95 \% \mathrm{CI}[1.17,2.25])$ and control conditions $(\mathrm{t}(87)=4.02, p<.001,95 \% \mathrm{CI}[0.55,1.62])$ showed significantly increased CO relative to baseline (see Figure 3, Panel B).

Gratitude expressions $(M=-65.96, S D=236.13)$ generated marginally lower (more challenge-patterned) TPR reactivity relative to the control condition $(M=-3.88, S D=162.96)$, $F(1,85)=3.60, p=.060$. Whereas the gratitude condition produced challenge-patterned TPR reactivity that significantly differed from zero $(t(85)=-2.91, p=.005,95 \%$ CI $[-111.09,-$ $20.835])$, control condition TPR reactivity did not significantly differ from zero $(t(85)=-$ $0.16, p=.870$ ) (see Figure 3, Panel C).

Individual Performance Task: Controlling for PA. We conducted an ANCOVA to control for speakers' positive affect during manipulation, and did not find that PA could account for the observed effects. Gratitude dyads continued to produce more challenge-patterned physiological responses compared to the control condition, as measured by challenge-threat index reactivity, $F(1,153)=7.72, p=.006,95 \%$ CI $[0.14,0.78], d=.45$. Gratitude dyads also now showed significantly higher $\mathrm{CO}$ reactivity, $F(1,89)=4.43, p=.038,95 \% \mathrm{CI}[0.02,0.70], d$ $=.45$, and now showed significantly lower TPR reactivity, $F(1,153)=4.28, p=.040,95 \%$ CI $[-$ 0.67, -0.02], $d=-.33$, compared to control dyads when controlling for speakers' PA.

Exploratory analyses of effects on expressers versus receivers. The present experiment was designed and powered to test effects on dyads. However, we conducted additional exploratory tests of how the gratitude manipulation influenced expressers versus receivers. For the collaborative work task, there was no main effect of role (expresser vs. 
receiver) on challenge-threat index reactivity, $F(1,82)=0.50, p=.480$. Nor was there a significant condition $*$ role interaction, $F(1,82)=1.31, p=.256$. Similarly, for the individual performance task, there was no main effect of role (expresser vs. receiver) on challenge-threat index reactivity, $F(1,67)=1.69, p=.199$. Nor was there a significant condition $*$ role interaction, $F(1,67)=0.09, p=.767$. Thus, we did not find strong evidence that the gratitude manipulation influenced expressers and receivers differently. Analyses of how the gratitude manipulation impacted expressers and receivers separately are provided in the Supplemental Material; we do not interpret these analyses due to a lack of power.

\section{Discussion}

Building on evidence showing that gratitude builds social and psychological resources in members of romantic relationships (Algoe \& Zhaoyang, 2016), we anticipated that gratitude expressions would increase teammates' biological resources when faced with stressful tasks by eliciting more challenge-patterned physiological stress responses. This pattern of results would provide the first evidence that gratitude builds biological resources, promoting better stress responses. The present study significantly advanced the gratitude literature by proposing and testing whether gratitude expressions would enhance physiological stress responding, specifically, and by demonstrating these effects in an understudied population in the gratitude literature, teammates - all in real time. Using an ecologically-valid, stressful work task that increased sympathetic arousal for all participants, our hypotheses focused on efficiency in cardiovascular responding - that is, an improved challenge-threat physiological stress response profile. As predicted, teammates showed improved challenge-threat responding as measured by the challenge-threat index, compared to controls, when one member of the team expressed gratitude to the other in a laboratory-based conversation prior to engaging in demanding tasks. 
These effects were observed at two crucial time points: (1) when the teammates were working together collaboratively to develop a product pitch, and (2) later when they independently pitched their part of the project to stoic evaluators. During the collaborative task, gratitude expressions buffered against threat responses, as indicated by the focal challenge-threat index. The control condition produced threat-patterned TPR reactivity that significantly differed from zero, whereas gratitude condition TPR reactivity was only marginally different from zero. In contrast, during the individual task, gratitude expressions amplified challenge responses, as indicated by the focal challenge-threat index. Controls showed a modest challenge response driven by myocardial influence (i.e., increased cardiac outflow) but no change in TPR, whereas gratitude expressions facilitated a challenge response consisting of both increased cardiac outflow and improved vascular response.

Importantly, both the gratitude and control conditions involved engaging in collaborative teamwork with a familiar, loose tie teammate. Further, follow-up analyses found that the expresser's positive affect during the manipulation could not account for the observed effects: When controlling for speaker's PA, the gratitude condition produced significantly improved values on the challenge-threat index, CO, and TPR during both the collaborative and individual tasks. Thus, the expression of gratitude, rather than positive affect or social support from the presence of a known other, drove the observed effects on challenge and threat. These findings substantially contribute to the gratitude literature, which has largely not produced evidence regarding physiology, nor about loose-tie social relationships (e.g., acquaintances or coworkers), which represent an important aspect of life. This work also adds an important theoretical and empirical twist in the consideration of relationship partners as resources during physiologically taxing episodes. 


\section{Physiological consequences of expressed gratitude}

Several studies document psychosocial consequences of expressed gratitude for the person who expresses it and for the person toward whom it is directed: Gratitude expressions are an inherently dyadic experience. Because the central benefit of these interactions relates to improved relationship quality (Algoe, 2012), and interpersonal relationships serve as resources to help people get through stressful times (Beckes \& Coan, 2011; Coan, Schaefer, \& Davidson, 2006; Cohen \& Wills, 1985; Conner et al., 2012; Page-Gould et al., 2014), we reasoned that an expression of gratitude would facilitate physiological resilience - in the form of improved challenge-threat physiological responses_-during a stressful task. These findings are the first of which we are aware to document physiological consequences from interpersonal gratitude. Critically, improved cardiovascular responses represent a meaningful consequence with potential translation to the challenges people face in their everyday lives.

The first finding — that gratitude buffered against biological threat responses during collaborative teamwork, as measured by the challenge-threat index-is important because this context models acutely stressful collaborative work typical of loose-tie teams within organizations. These findings represent the first evidence of gratitude's impact on biological stress - research thus far has shown that dispositional gratitude is related to subjective stress (Deutsch, 1984; Krause, 2006) and helps decrease subjective stress over time (Wood et al., 2008) — as well as the first evidence of gratitude's impact on stress processes in members of dyads or teams. The second finding - that gratitude expressions enhanced cardiovascular efficiency later when individuals completed an individual performance task-is distinctly important for three reasons. First, the two teammates did not directly interact during the individual performance task; thus, direct interaction between participants was not necessary for 
gratitude's positive impact on biological stress responding to persist. Second, the individual performance task occurred approximately 12 minutes after the conclusion of the gratitude manipulation (in contrast to the collaborative work task, which occurred directly afterward); this indicates that the gratitude manipulation influenced physiological responses for at least this period of time. Third, the individual performance task was modeled on the Trier Social Stress Task (Kirschbaum et al., 1993) allowing a direct comparison to how other studies' manipulations impact stress responding for individuals in the same context.

\section{Biopsychosocial model of challenge and threat}

The present research was grounded in the BPS model of challenge and threat, which sheds light on the biological mechanisms underlying how people respond to stress (Blascovich \& Mendes, 2010). Gratitude expressions improved cardiovascular efficiency in the expresserreceiver dyad — facilitating delivery of oxygenated blood to the periphery and brain-in two distinct contexts: when collaborating, and later when working individually. In addition, demonstrating the physiological benefits of a simple gratitude expression in a team performance task has potentially broader implications because, relative to threat responses, challenge responses are correlated with reduced attention to negative cues (Jamieson et al., 2012), facilitating decision making (Kassam et al., 2009), slower "brain aging” (Jefferson et al., 2010), and predict academic success (Seery et al., 2010). The current study is the first to directly investigate the immediate and subsequent consequences of gratitude expression on acute stress in a dyadic team performance context.

The present work also informs challenge and threat theory by demonstrating that not only can emotion regulatory activities modulate challenge and threat responses in team performance contexts (Oveis et al., 2020), but also that emotion expressions (specifically, gratitude) and 
interpersonal dynamics can facilitate stress responses in the body. This has important implications in that it suggests potential interventions that can change the perception of one's resources versus contextual demands, thus increasing challenge states and potentially boosting task performance.

\section{Gratitude among loose social ties}

Whereas important work has been conducted on gratitude between strangers and romantic partners, a novel area of interest relates to gratitude in the workplace (Fehr, Fulmer \& Miller, 2017). Adults often spend the majority of the waking day at work, engaging in social interactions within networks of looser social ties. However, few studies have examined gratitude in this important relational context (e.g., Lee et al., 2019), and none look closely at the dyad or the consequences of gratitude in vivo. Despite the documented benefits of expressing gratitude on strengthening social bonds (Algoe et al., 2020), many people are reluctant to express gratitude because they fear that others will not appreciate their expressions (Kumar \& Epley, 2018), or perhaps fearing a loss of status in others' eyes (Chaudhry \& Loewenstein, 2019). This reluctance may be exacerbated in professional settings, and research demonstrating the impact of gratitude in loose-tie teams provides an empirical basis for expressing more gratitude in the workplace. The present research presents an important methodological tool for use in future gratitude research, by presenting an ecologically valid paradigm to study gratitude's impact on teamwork and stress responding, and by focusing on resilient physiological profiles of challenge vs. threat responses.

\section{Limitations}

The following limitations should be considered in interpreting the present findings. First, even though the teammates in the present study are newly acquainted suitemates living in the 
same dorm, these relationships are not strictly representative of work teammates. The present research, however, suggests that work with professional teammates would be fruitful. Second, the present study employed an experimental manipulation of gratitude expressions; future work should examine individual differences in gratitude, and determine if adding a person to a team who tends to express gratitude would produce team-level benefits. Third, with the rise of virtual teamwork, we note that the gratitude expression and positive impact of stress-responding in teams occurred in a face-to-face setting. We speculate that gratitude expressions would exert similar effects when expressed via a technological medium, but future research is necessary to support this claim. Finally, regarding the individual task, we note that the task did not involve direct interaction between teammates, and the performing teammate glanced at their partner in very few instances. However, the two teammates did sit next to one another, which raises the possibility of social influence impacting the results. And, indeed, social baseline theory suggests that the presence of others reduces threat-related neural activity (Beckes \& Coan, 2011). It is possible that having the participants perform the task physically separated from their partner would produce different results, but this is a possibility that remains to be tested.

\section{Conclusion}

The present findings provide the first evidence that gratitude expressions impact biological responses in teammates, for the better. This work fits with a burgeoning literature on the social consequences of gratitude (e.g., Algoe et al., 2020), and more generally with work suggesting a myriad of positive intra- and interpersonal consequences of positive interpersonal processes (Algoe, 2019). The evidence here suggests a potential benefit of injecting gratitude into teams and organizations: One person's gratitude can positively impact a team at a biological level and promote more adaptive responses to stress. 


\section{References}

Algoe, S. (2012). Find, remind, and bind: The functions of gratitude in everyday relationships. Social and Personality Psychology Compass, 6, 455-469. https://doi.org/10.1111/j.17519004.2012.00439.x

Algoe, S. B. (2019). Positive interpersonal processes. Current Directions in Psychological Science, 28(2), 183-188. https://doi.org/10.1177/0963721419827272

Algoe, S. B., Dwyer, P. C., Younge, A., \& Oveis, C. (2020). A new perspective on the social functions of emotions: Gratitude and the witnessing effect. Journal of Personality and Social Psychology, 119(1), 40-74. https://doi.org/10.1037/pspi0000202

Algoe, S. B., Fredrickson, B. L., \& Gable, S. L. (2013). The social functions of the emotion of gratitude via expression. Emotion, 13(4), 605-609. https://doi.org/10.1037/a0032701

Algoe, S. B., \& Zhaoyang, R. (2016). Positive psychology in context: Effects of expressing gratitude in ongoing relationships depend on perceptions of enactor responsiveness. The Journal of Positive Psychology, 11(4), 399-415. https://doi.org/10.1080/17439760.2015.1117131

Beckes, L., \& Coan, J. A. (2011). Social baseline theory: The role of social proximity in emotion and economy of action. Social and Personality Psychology Compass, 5(12), 976-988. https://doi.org/10.1111/j.1751-9004.2011.00400.x

Blascovich J., Mendes W. B. (2000). Challenge and threat appraisals: The role of affective cues.. In J. P. Forgas (Ed.), Feeling and thinking: The role of affect in social cognition (pp. 5982). Cambridge, UK: Cambridge University Press.

Blascovich, J., \& Mendes, W. B. (2010). Social psychophysiology and embodiment. In Handbook of social psychology, Vol. 1, 5th ed (pp. 194-227). John Wiley \& Sons Inc. 
Blascovich, J., Seery, M. D., Mugridge, C. A., Norris, R. K., \& Weisbuch, M. (2004). Predicting athletic performance from cardiovascular indexes of challenge and threat. Journal of Experimental Social Psychology, 40(5), 683-688. https://doi.org/10.1016/j.jesp.2003.10.007

Blascovich, J., \& Tomaka, J. (1996). The biopsychosocial model of arousal regulation. In Advances in experimental social psychology (Vol. 28, pp. 1-51). Academic Press.

Blascovich, J., Vanman, E., Mendes, W. B., \& Dickerson, S. (2011). Social psychophysiology for social and personality psychology. Sage Publications.

Brady, A., Baker, L. R., Muise, A., \& Impett, E. A. (2020). Gratitude increases the motivation to fulfill a partner's sexual needs. Social Psychological and Personality Science, 1948550619898971. https://doi.org/10.1177/1948550619898971

Chaudhry, S. J., \& Loewenstein, G. (2019). Thanking, apologizing, bragging, and blaming: Responsibility exchange theory and the currency of communication. Psychological Review, 126(3), 313-344. https://doi.org/10.1037/rev0000139

Coan, J. A., Schaefer, H. S., \& Davidson, R. J. (2006). Lending a hand: Social regulation of the neural response to threat. Psychological Science, 17(12), 1032-1039. https://doi.org/10.1111/j.1467-9280.2006.01832.x

Cohen, S., \& Wills, T. A. (1985). Stress, social support, and the buffering hypothesis. Psychological Bulletin, 98(2), 310-357. https://doi.org/10.1037/0033-2909.98.2.310

Conner, O. L., Siegle, G. J., McFarland, A. M., Silk, J. S., Ladouceur, C. D., Dahl, R. E., Coan, J. A., \& Ryan, N. D. (2012). Mom-it helps when you're right here! Attenuation of neural stress markers in anxious youths whose caregivers are present during fMRI. PloS One, 7(12), e50680. https://doi.org/10.1371/journal.pone.0050680 
Deutsch, C. J. (1984). Self-reported sources of stress among psychotherapists. Professional Psychology: Research and Practice, 15(6), 833-845. https://doi.org/10.1037/07357028.15.6.833

Drążkowski, D., Kaczmarek, L., \& Kashdan, T. (2017). Gratitude pays: A weekly gratitude intervention influences monetary decisions, physiological responses, and emotional experiences during a trust-related social interaction. Personality and Individual Differences, 110, 148-153. https://doi.org/10.1016/j.paid.2017.01.043

Fehr, R., Fulmer, A., Awtrey, E., \& Miller, J. A. (2017). The grateful workplace: A multilevel model of gratitude in organizations. Academy of Management Review, 42(2), 361-381. https://doi.org/10.5465/amr.2014.0374

Ginty, A. T., Tyra, A. T., Young, D. A., John-Henderson, N. A., Gallagher, S., \& Tsang, J.-A. C. (2020). State gratitude is associated with lower cardiovascular responses to acute psychological stress: A replication and extension. International Journal of Psychophysiology, 158, 238-247. https://doi.org/10.1016/j.ijpsycho.2020.10.005

Griffin, S. M., \& Howard, S. (in press). Individual differences in emotion regulation and cardiovascular responding to stress. Emotion.

Griffin, S. M., \& Howard, S. (2021). Instructed reappraisal and cardiovascular habituation to recurrent stress. Psychophysiology, 58(5), e13783.

Jamieson, J. P., Mendes, W. B., \& Nock, M. K. (2013). Improving acute stress responses: The power of reappraisal. Current Directions in Psychological Science, 22(1), 51-56. https://doi.org/10.1177/0963721412461500 
Jefferson, A. L., Himali, J. J., Beiser, A. S., Au, R., Massaro, J. M., Seshadri, S., ... Manning, W. J. (2010). Cardiac index is associated with brain aging: The Framingham Heart Study. Circulation, 122(7), 690-697. doi:10.1161/circulationaha.109.905091

Kassam, K. S., Koslov, K., \& Mendes, W. B. (2009). Decisions under distress: Stress profiles influence anchoring and adjustment. Psychological Science, 20(11), 1394-1399. doi:10.1111/j.1467-9280.2009.02455.x

Kirschbaum, C., Pirke, K. M., \& Hellhammer, D. H. (1993). The 'Trier Social Stress Test'—A tool for investigating psychobiological stress responses in a laboratory setting. Neuropsychobiology, 28(1-2), 76-81. https://doi.org/10.1159/000119004

Krause, N. (2006). Gratitude toward God, stress, and health in late life. Research on Aging - RES AGING, 28, 163-183. https://doi.org/10.1177/0164027505284048

Kumar, A., \& Epley, N. (2018). Undervaluing gratitude: Expressers misunderstand the consequences of showing appreciation: Psychological Science, 29, 1423-1435. https://doi.org/10.1177/0956797618772506

Lee, H. W., Bradburn, J., Johnson, R. E., Lin, S.-H. (J), \& Chang, C.-H. (D). (2019). The benefits of receiving gratitude for helpers: A daily investigation of proactive and reactive helping at work. Journal of Applied Psychology, 104(2), 197-213. https://doi.org/10.1037/ap10000346

Leong, J. L. T., Chen, S. X., Fung, H. H. L., Bond, M. H., Siu, N. Y. F., \& Zhu, J. Y. (2020). Is Gratitude always beneficial to interpersonal relationships? The interplay of grateful disposition, grateful mood, and grateful expression among married couples. Personality and Social Psychology Bulletin, 46(1), 64-78. https://doi.org/10.1177/0146167219842868 
Lundberg, U. (2005). Stress hormones in health and illness: The roles of work and gender. Psychoneuroendocrinology, 30(10), 1017-1021. doi:10.1016/j.psyneuen.2005.03.014

Matthews, K. A., Gump, B. B., Block, D. R., \& Allen, M. T. (1997). Does background stress heighten or dampen children's cardiovascular responses to acute stress? Psychosomatic Medicine, 59(5), 488-496. doi:10.1016/j.psyneuen.2005.03/014

Mendes, W. B., \& Park, J. (2014). Chapter six-Neurobiological concomitants of motivational states. In A. J. Elliot (Ed.), Advances in Motivation Science (Vol. 1, pp. 233-270). Elsevier. https://doi.org/10.1016/bs.adms.2014.09.001

Moore, L. J., Vine, S. J., Wilson, M. R., \& Freeman, P. (2012). The effect of challenge and threat states on performance: An examination of potential mechanisms. Psychophysiology, 49(10), 1417-1425. https://doi.org/10.1111/j.1469-8986.2012.01449.x

Naschitz, J. E., Gaitini, L., Loewenstein, L., Keren, D., Zuckerman, E., Tamir, A., \& Yeshurun, D. (2000). In-field validation of automatic blood pressure measuring devices. Journal of Human Hypertension, 14(1), 37-42. https://doi.org/10.1038/sj.jhh.1000937

Oveis, C., Gu, Y., Ocampo, J. M., Hangen, E. J., \& Jamieson, J. P. (2020). Emotion regulation contagion: Stress reappraisal promotes challenge responses in teammates. Journal of Experimental Psychology. General, 149, 2187-2205. https://doi.org/10.1037/xge0000757

Page-Gould, E., Mendoza-Denton, R., \& Mendes, W. B. (2014). Stress and coping in interracial contexts: The influence of race-based rejection sensitivity and cross-group friendship in daily experiences of health. The Journal of Social Issues, 70(2), 256-278. https://doi.org/10.1111/josi.12059

Park, Y., Impett, E. A., MacDonald, G., \& Lemay, E. P. (2019). Saying “thank you”: Partners' expressions of gratitude protect relationship satisfaction and commitment from the 
harmful effects of attachment insecurity. Journal of Personality and Social Psychology, 117(4), 773-806. https://doi.org/10.1037/pspi0000178

Peters, B. J., Overall, N. C., \& Jamieson, J. P. (2014). Physiological and cognitive consequences of suppressing and expressing emotion in dyadic interactions. International Journal of Psychophysiology, 94(1), 100-107. doi:10.1016/j.ijpsycho.2014.07.015

Raudenbush, S. W., et al. (2011). Optimal Design Software for Multi-level and Longitudinal Research (Version 3.01) [Software]. Available from www.wtgrantfoundation.org.

Redwine, L. S., Henry, B. L., Pung, M. A., Wilson, K., Chinh, K., Knight, B., Jain, S., Rutledge, T., Greenberg, B., Maisel, A., \& Mills, P. J. (2016). Pilot randomized study of a gratitude journaling intervention on heart rate variability and inflammatory biomarkers in patients with stage B heart failure. Psychosomatic Medicine, 78(6), 667-676.

https://doi.org/10.1097/PSY.0000000000000316

Schnall, S., Harber, K. D., Stefanucci, J. K., \& Proffitt, D. R. (2008). Social support and the perception of geographical slant. Journal of Experimental Social Psychology, 44(5), 1246-1255. https://doi.org/10.1016/j.jesp.2008.04.011

Seery, M. D. (2011). Challenge or threat? Cardiovascular indexes of resilience and vulnerability to potential stress in humans. Neuroscience and Biobehavioral Reviews, 35(7), 16031610. https://doi.org/10.1016/j.neubiorev.2011.03.003

Seery, M. D. (2013). The biopsychosocial model of challenge and threat: Using the heart to measure the mind. Social and Personality Psychology Compass, 7(9), 637-653. https://doi.org/10.1111/spc3.12052 
Seery, M. D., Weisbuch, M., Hetenyi, M. A., \& Blascovich, J. (2010). Cardiovascular measures independently predict performance in a university course. Psychophysiology, 47(3), 535539. https://doi.org/10.1111/j.1469-8986.2009.00945.x

Turner, M. J., Jones, M. V., Sheffield, D., \& Cross, S. L. (2012). Cardiovascular indices of challenge and threat states predict competitive performance. International Journal of Psychophysiology: Official Journal of the International Organization of Psychophysiology, 86(1), 48-57. https://doi.org/10.1016/j.ijpsycho.2012.08.004

Williams, L. A., \& Bartlett, M. Y. (2015). Warm thanks: Gratitude expression facilitates social affiliation in new relationships via perceived warmth. Emotion, 15(1), 1-5. https://doi.org/10.1037/emo0000017

Wood, A. M., Maltby, J., Gillett, R., Linley, P. A., \& Joseph, S. (2008). The role of gratitude in the development of social support, stress, and depression: Two longitudinal studies. Journal of Research in Personality, 42(4), 854-871. https://doi.org/10.1016/j.jrp.2007.11.003 